\title{
A DIVERGENT KHINTCHINE THEOREM IN THE REAL, COMPLEX, AND $p$-ADIC FIELDS
}

\author{
V. Bernik ${ }^{1}$, N. Budarina ${ }^{2}$ and D. Dickinson ${ }^{2}$ \\ ${ }^{1}$ Institute of Mathematics, National Academy of Sciences of Belarus, Surganova 11, 220072 Minsk, Belarus \\ (e-mail: bernik@im.bas-net.by) \\ ${ }^{2}$ Department of Mathematics, National University of Ireland, Maynooth, Co. Kildare, Republic of Ireland \\ (e-mail: buda77@mail.ru; ddickinson@maths.nuim.ie)
}

Received October 24, 2007

\begin{abstract}
In this paper, we show that if the sum $\sum_{r=1}^{\infty} \Psi(r)$ diverges, then the set of points $(x, z, w) \in \mathbb{R} \times \mathbb{C} \times \mathbb{Q}_{p}$ satisfying the inequalities $|P(x)|<H^{-v_{1}} \Psi^{\lambda_{1}}(H),|P(z)|<H^{-v_{2}} \Psi^{\lambda_{2}}(H)$, and $|P(w)|_{p}<H^{-v_{3}} \Psi^{\lambda_{3}}(H)$ for infinitely many integer polynomials $P$ has full measure. With a special choice of parameters $v_{i}$ and $\lambda_{i}, i=1,2,3$, we can obtain all the theorems in the metric theory of transcendental numbers which were known in the real, complex, or $p$-adic fields separately.
\end{abstract}

Keywords: Diophantine approximation, Khintchine-type theorems, metric theory of transcendental numbers.

A starting point for many problems in metric Diophantine approximation is Khintchine's theorem [10] proved more than 80 years ago. Let $\Psi(x)$ be a decreasing function defined for $x>0$, and let $\mu_{1}(A)$ denote the Lebesgue measure of a measurable set $A \subset \mathbb{R}$. Let $I \subset \mathbb{R}$ be an interval. The set $L_{1}(\Psi)$ is defined as the set of real numbers $x \in I$ satisfying the inequality

$$
|x-p / q|<\Psi(q) / q
$$

for infinitely many $p, q \in \mathbb{Z}$ with $q \neq 0$.

Theorem 1 [Khintchine].

$$
\mu_{1} L_{1}(\Psi)= \begin{cases}0, & \sum_{r=1}^{\infty} \Psi(r)<\infty \\ \mu_{1}(I), & \sum_{r=1}^{\infty} \Psi(r)=\infty\end{cases}
$$

There are many extensions and generalizations of this theorem, and this paper is concerned with one of them. In [8], a convergent Khintchine theorem was obtained for the approximation of zero by integer polynomials in the real, complex, and $p$-adic fields simultaneously. In this paper, we consider the divergence part. First, let

$$
P(f)=a_{n} f^{n}+a_{n-1} f^{n-1}+\ldots+a_{1} f+a_{0}, \quad a_{j} \in \mathbb{Z},
$$


be an $n$th degree integer polynomial with height $H=H(P)=\max _{1 \leqslant j \leqslant n}\left|a_{j}\right|$. Using Dirichlet's box principle or Minkowski's linear forms theorem, it is easy to show that, for $w \leqslant n$, the inequality

$$
|P(f)|<H^{-w}
$$

has infinitely many solutions $P \in \mathbb{Z}[x]$ (with $\operatorname{deg} P \leqslant n$ ) for all $x \in \mathbb{R}$. On the other hand, Sprindzuk [12] proved that, for almost all $x$, inequality (1) has at most finitely many solutions when $w>n$. We define $L_{n}(w)$ as the set of points $x \in \mathbb{R}$ for which the inequality

$$
|P(x)|<H^{-n+1} \Psi(H)
$$

has infinitely many solutions $P \in \mathbb{Z}[x]$. A full analogue of Khintchine's theorem for $L_{n}(w)$ can be found in $[5,1]$. This result has also been generalized to the field of complex numbers in [6] and to the field of $p$-adic numbers in $[11,3]$. In this paper, we generalize the divergence case to $S=\mathbb{R} \times \mathbb{C} \times \mathbb{Q}_{p}$.

To this end, let $\mu_{1}(A)$ be the Lebesgue measure of a measurable set $A \subset \mathbb{R}, \mu_{2}(A)$ the Lebesgue measure of a measurable set $A \subset \mathbb{C}$, and $\mu_{3}(A)$ the Haar measure of a measurable set $A \subset \mathbb{Q}_{p}$. We put these together in the obvious way by defining $\mu(A)=\mu(B \times C \times D)=\mu_{1}(B) \mu_{2}(C) \mu_{3}(D)$ for a measurable set $A=B \times C \times D \subset \mathbb{R} \times \mathbb{C} \times \mathbb{Q}_{p}$. Let $\mathbf{v}=\left(v_{1}, v_{2}, v_{3}\right)$ and $\boldsymbol{\lambda}=\left(\lambda_{1}, \lambda_{2}, \lambda_{3}\right)$ be real vectors satisfying $v_{1}, v_{2} \geqslant-\frac{1}{4}, v_{3} \geqslant 0$, and $\lambda_{i}>0, i=1,2,3$. Denote by $\mathcal{L}_{\mathbf{v}, \boldsymbol{\lambda}}(\Psi)$ the set of points in a parallelepiped $T=I \times K \times D$, where $I \subset \mathbb{R}$ is an interval, $K \subset \mathbb{C}$ is a disk, and $D \subset \mathbb{Q}_{p}$ is a cylinder, for which the system of inequalities

$$
\begin{aligned}
& |P(x)|<H^{-v_{1}} \Psi^{\lambda_{1}}(H), \\
& |P(z)|<H^{-v_{2}} \Psi^{\lambda_{2}}(H), \\
& |P(w)|_{p}<H^{-v_{3}} \Psi^{\lambda_{3}}(H),
\end{aligned}
$$

with $v_{1}+2 v_{2}+v_{3}=n-3$ and $\lambda_{1}+2 \lambda_{2}+\lambda_{3}=1$ has infinitely many solutions $P \in \mathbb{Z}[x]$. In this paper, we prove the following theorem.

Theorem 2. Suppose that (2) holds. If $n \geqslant 3$ and $\sum_{r=1}^{\infty} \Psi(r)=\infty$, then

$$
\mu\left(L_{\mathbf{v}, \boldsymbol{\lambda}}(\Psi)\right)=\mu(T)
$$

with $\mathbf{v}=\left(\frac{n-4}{4}, \frac{n-4}{4}, \frac{n}{4}\right)$ and $\boldsymbol{\lambda}=\left(\frac{1}{4}, \frac{1}{4}, \frac{1}{4}\right)$.

Let $\delta_{1}>0$ be a fixed real number; without loss of generality we assume that points $z \in K$ satisfy $|\operatorname{Im} z| \geqslant \delta_{1}$. In what follows, inequalities of the form $|z-\alpha|<H(P)^{-t}, t>0$, appear several times. Since the RHS (the right-hand side) tends to zero as $H \rightarrow \infty$, it follows that (for sufficiently large $H$ ) $|\operatorname{Im} \alpha|>\frac{1}{2} \delta_{1}$. There is also a conjugate root $\bar{\alpha}$ of $P$ which clearly satisfies $|\alpha-\bar{\alpha}|>\delta_{1}$. Similarly, for a real $\operatorname{root} \beta$ of $P$, the inequalities $|\beta-\alpha|=|\beta-\bar{\alpha}|>\frac{1}{2} \delta_{1}$ hold. Hence, from now on we assume that

$$
\begin{aligned}
& |\operatorname{Im} \alpha|>\frac{1}{2} \delta_{1}, \\
& |\operatorname{Im} z| \geqslant \delta_{1}, \\
& |\alpha-\bar{\alpha}|>\delta_{1}, \\
& |\beta-\alpha|>\frac{1}{2} \delta_{1} .
\end{aligned}
$$

Given the conditions of Theorem 2 and (3), the restriction $\operatorname{deg} P \geqslant 3$ is automatic.

One of the main parts of the proof of this theorem is the construction of an optimal regular system of sets of roots of integer polynomials $P$ of the form $(\alpha, \beta, \gamma), P(\alpha)=P(\beta)=P(\gamma)=0$ with $\alpha \in \mathbb{R}, \beta \in \mathbb{C}$, 
$|\operatorname{Im} \beta|>\frac{1}{2} \delta_{1}$, and $\gamma \in \mathbb{Q}_{p}$. In [2], an optimal regular system is defined and is then used to prove a divergent Khintchine theorem. We shall give a similar obvious construction of an optimal regular system for the roots discussed above.

Let $P \in \mathbb{Z}[x]$ be an irreducible polynomial over $\mathbb{R}$ with height $H=H(P)$ and roots $\alpha \in \mathbb{R}, \beta \in$ $\mathbb{C}$, and $\gamma \in \mathbb{Q}_{p}$. Assume that $P$ is the minimal polynomial for $\alpha, \beta$, and $\gamma$. Consider a vector $\mathbf{w}=$ $\left(w_{1}+1, w_{2}+1, w_{3}\right)$ such that

$$
w_{1}, w_{2} \geqslant 0, \quad w_{3}>0, \quad w_{1}+2 w_{2}+w_{3}=n-2 .
$$

DEFINITION 1. The set of points $\boldsymbol{\nu}=(\alpha, \beta, \gamma)$ with the vector-function

$$
N(\boldsymbol{\nu})=\left(H^{w_{1}+1}, H^{w_{2}+1}, H^{w_{3}}\right)
$$

is called an optimal regular system if there exist constants $c_{1}>0$ and $c_{2}>0$ such that, for any parallelepiped $T_{0} \subset T$, there exists a sufficiently large number $M_{0}\left(T_{0}\right)$ such that, for any integer $M>M_{0}$, we can choose points $\boldsymbol{\nu}_{1}, \boldsymbol{\nu}_{2}, \cdots, \boldsymbol{\nu}_{t}$ such that:

$$
H^{n+1}(\alpha)<M
$$

the parallelepipeds

$$
\Pi_{i}=\left\{\left|x-\alpha_{i}\right|<M^{-w_{1}-1},\left|z-\beta_{i}\right|<M^{-w_{2}-1},\left|w-\gamma_{i}\right|_{p}<M^{-w_{3}}\right\}
$$

do not intersect for any $i, j, 1 \leqslant i<i<j \leqslant t$, and

$$
c_{1} M \mu\left(T_{0}\right)<t<c_{2} M \mu\left(T_{0}\right) .
$$

If (5) satisfies $t>c_{1} M \mu\left(T_{0}\right)$, then the system is called a regular system.

We use $c(n)$ to denote positive constants depending only on $n$; we use the formal rules $c(n)+c(n)=c(n)$ and $c(n) c(n)=c(n)$. Where necessary, the constants will be indexed $c_{j}, j=1,2, \ldots$.

Using Dirichlet's box principle, it is easy to prove that, for some constant $c_{3}>0$, any point $\mathbf{u} \in T_{0}$, for any $Q>1$, and for numbers $w_{1}, w_{2}, w_{3}$ such that $w_{1}+2 w_{2}+w_{3} \leqslant n-2$, we can find a nonzero polynomial $P$ such that

$$
\begin{aligned}
& |P(x)|<c_{3} Q^{-w_{1}}, \\
& |P(z)|<c_{3} Q^{-w_{2}}, \\
& |P(w)|_{p}<c_{3} Q^{-w_{3}},
\end{aligned}
$$

with $H(P) \leqslant Q$ and $\operatorname{deg} P \leqslant n$. Clearly, it is necessary to consider $w_{3}>0$.

The system of inequalities (6) forms a base for construction of an optimal regular system of roots of polynomials $P \in \mathbb{Z}[x]$ which are close to $x, z$, and $w$, respectively.

Theorem 3. For any vector $\mathbf{w}=\left(w_{1}, w_{2}, w_{3}\right)$ satisfying (4), it is possible to construct a regular system from the vectors $\nu$.

The important part of this is the simultaneous solvability of system (6) and the system

$$
\begin{aligned}
& \left|P^{\prime}(x)\right|>c_{5} Q, \\
& \left|P^{\prime}(z)\right|>c_{5} Q, \\
& \left|P^{\prime}(w)\right|_{p}>c_{5},
\end{aligned}
$$


for some set $B_{0} \subset T_{0}$ with $\mu\left(B_{0}\right)>\mu\left(T_{0}\right) / 2$ and some constant $c_{5}>0$. There are several cases to consider by replacing one or more of the $>$ signs with $\leqslant$ in the above system.

For some constant $\epsilon>0$, denote by $B_{11}$ the set of $\mathbf{u} \in T_{0}$ for which (6) and

$$
\begin{aligned}
& Q^{1 / 2}<\left|P^{\prime}(x)\right| \leqslant c_{5} Q \\
& Q^{1 / 2}<\left|P^{\prime}(z)\right| \leqslant c_{5} Q \\
& Q^{-\epsilon}<\left|P^{\prime}(w)\right|_{p} \leqslant c_{5}
\end{aligned}
$$

are satisfied simultaneously. We will prove that there exists a constant $c_{5}^{\prime}$ such that $\mu\left(B_{11}\right)<2^{-6} \mu\left(T_{0}\right)$ for $c_{5}<c_{5}^{\prime}$.

Let $\alpha_{1}, \alpha_{2}, \cdots, \alpha_{n}$ be the complex roots of $P$ and $\gamma_{1}, \gamma_{2}, \cdots, \gamma_{n}$ be the roots of $P$ in $\mathbb{Q}_{p}^{*}$, where $\mathbb{Q}_{p}^{*}$ is the smallest field containing $\mathbb{Q}_{p}$ and all algebraic numbers. Suppose, without loss of generality, that all the coordinates of $\mathbf{u}$ are transcendental (the measure of $\mathbf{u} \in \mathbb{R} \times \mathbb{C} \times \mathbb{Q}_{p}$ that have at least one algebraic coordinate is zero). It is also possible to assume that the polynomials in (2) are irreducible and that they satisfy the conditions

$$
H=H(P)=\left|a_{n}\right|, \quad\left|a_{n}\right|_{p}>p^{-n} .
$$

The reduction of the general case to irreducible polynomials is done in [12]. Let $P_{n}(H)$ be the set of irreducible polynomials $P$ satisfying the conditions with $\operatorname{deg} P \leqslant n$. Further define $P_{n}=\bigcup_{H=1}^{\infty} P_{n}(H)$. It is not difficult also to obtain that

$$
\left|\alpha_{i}\right| \leqslant 2 \text { and }\left|\gamma_{i}\right|<p^{n}
$$

for $i=1, \ldots, n$. Order the roots $\alpha_{i}, 1 \leqslant i \leqslant n$, of $P \in P_{n}(H)$ by increasing real part; if two roots have equal real parts, then order by increasing nonnegative imaginary part. The roots $\gamma_{i}, 1 \leqslant i \leqslant n$, are ordered by increasing norm. For every root $\alpha_{i}, \gamma_{i}, 1 \leqslant i \leqslant n$, define the sets

$$
\begin{aligned}
& S\left(\alpha_{i}\right)=\left\{z \in \mathbb{C}:\left|z-\alpha_{i}\right|=\min _{1 \leqslant j \leqslant n}\left|z-\alpha_{j}\right|\right\}, \\
& S^{\prime}\left(\alpha_{i}\right)=S\left(\alpha_{i}\right) \cap \mathbb{R}, \\
& S_{p}\left(\gamma_{i}\right)=\left\{w \in \mathbb{Q}_{p}:\left|w-\gamma_{i}\right|_{p}=\min _{1 \leqslant j \leqslant n}\left|w-\gamma_{j}\right|_{p}\right\} .
\end{aligned}
$$

Clearly, we can fix $j$ and consider the system of inequalities with vectors $\mathbf{u} \in S^{\prime}\left(\alpha_{i}\right) \times S\left(\alpha_{j}\right) \times S\left(\gamma_{k}\right)$, $1 \leqslant i, j, k \leqslant n$. Without loss of generality, we shall suppose that $i=j=k=1$ and order the remaining roots with respect to $\alpha_{1}, \beta_{1}$, and $\gamma_{1}$ so that

$$
\begin{aligned}
& \left|\alpha_{1}-\alpha_{2}\right| \leqslant\left|\alpha_{1}-\alpha_{3}\right| \leqslant \ldots \leqslant\left|\alpha_{1}-\alpha_{n_{1}}\right|, \\
& \left|\beta_{1}-\beta_{2}\right| \leqslant\left|\beta_{1}-\beta_{3}\right| \leqslant \ldots \leqslant\left|\beta_{1}-\beta_{n_{2}}\right|, \\
& \left|\gamma_{1}-\gamma_{2}\right|_{p} \leqslant\left|\gamma_{1}-\gamma_{3}\right|_{p} \leqslant \ldots \leqslant\left|\gamma_{1}-\gamma_{n}\right|_{p}
\end{aligned}
$$

with $n_{1}+n_{2}=n$.

The next lemma is proved in [12]. 
Lemma 1. Let $P \in P_{n}(H)$ and $x \in S_{1}\left(\alpha_{1}\right), z \in S_{2}\left(\alpha_{1}\right), w \in S_{p}\left(\gamma_{1}\right)$. Then

$$
\begin{aligned}
& \left|u-\xi_{1}\right| \leqslant 2^{n}|P(u)|\left|P^{\prime}\left(\xi_{1}\right)\right|^{-1}, \\
& \left|w-\gamma_{1}\right| \leqslant|P(w)|_{p}\left|P^{\prime}\left(\gamma_{1}\right)\right|_{p}^{-1}, \\
& \left|u-\xi_{1}\right| \leqslant \min _{2 \leqslant j \leqslant n}\left(2^{n-j}|P(u)|\left|P^{\prime}\left(\xi_{1}\right)\right|^{-1} \prod_{k=2}^{j}\left|\xi_{1}-\xi_{k}\right|\right)^{\frac{1}{j}}, \\
& \left|w-\gamma_{1}\right|_{p} \leqslant \min _{2 \leqslant j \leqslant n}\left(|P(w)|_{p}\left|P^{\prime}\left(\gamma_{1}\right)\right|_{p}^{-1} \prod_{k=2}^{j}\left|\gamma_{1}-\gamma_{k}\right|_{p}\right)^{\frac{1}{j}},
\end{aligned}
$$

where $\left(u, \xi_{j}\right)=\left(x, \alpha_{j}\right)$ or $\left(z, \beta_{j}\right)$.

From (2), (9), (10), and Lemma 1 it follows that all solutions of (2) are contained in the parallelepiped $A=I_{1} \times K_{1} \times D_{1}$, where $I_{1}=[-3,3], K_{1}=\{z \in \mathbb{C}:|z| \leqslant 3\}$, and $D_{1}=\left\{w \in Q_{p},|w|_{p}<p^{n}\right\}$. Fix a sufficiently small number $10^{-3}>\epsilon>0$, and suppose that $\epsilon_{1}=\epsilon d^{-1}$, where $d=d(n)>0$ is sufficiently large.

We define the real numbers $\rho_{i j}, i=1,2,3,2 \leqslant j \leqslant n$, by

$$
\begin{aligned}
& \left|\alpha_{1}-\alpha_{j}\right|=H^{-\rho_{1 j}}, \\
& \left|\beta_{1}-\beta_{j}\right|=H^{-\rho_{2 j}}, \\
& \left|\gamma_{1}-\gamma_{j}\right|=H^{-\rho_{3 j}},
\end{aligned}
$$

and the integers $k_{j}, l_{j}, m_{j}$ for $2 \leqslant j \leqslant n$ from the relations

$$
\begin{aligned}
& \frac{k_{j}-1}{T} \leqslant \rho_{1 j}<\frac{k_{j}}{T}, \\
& \frac{l_{j}-1}{T} \leqslant \rho_{2 j}<\frac{l_{j}}{T}, \\
& \frac{m_{j}-1}{T} \leqslant \rho_{3 j}<\frac{m_{j}}{T} .
\end{aligned}
$$

Further, define the numbers $q_{i}, r_{i}, s_{i}(1 \leqslant i \leqslant n-1)$ by

$$
\begin{aligned}
q_{i} & =\frac{k_{i+1}+\ldots+k_{n}}{T}, \\
r_{i} & =\frac{l_{i+1}+\ldots+l_{n}}{T}, \\
s_{i} & =\frac{m_{i+1}+\ldots+m_{n}}{T} .
\end{aligned}
$$

We associate with each polynomial $P \in P_{n}(H)$ three integer vectors $\mathbf{q}=\mathbf{q}(P)=\left(k_{2}, \ldots, k_{n}\right), \mathbf{r}=\mathbf{r}(P)=$ $\left(l_{2}, \ldots, l_{n}\right)$, and $\mathbf{s}=\mathbf{s}(P)=\left(m_{2}, \ldots, m_{n}\right)$. From (10) it is possible to obtain lower bounds for $k_{j}, l_{j}$, and $m_{j}$ with $2 \leqslant j \leqslant n$. On the one hand, the discriminant of an irreducible polynomial is a natural number. On the other hand, the discriminant is determined by the product of the root differences. This means that the roots cannot be too close and therefore gives upper bounds for $k_{j}, l_{j}, m_{j}$ with $2 \leqslant j \leqslant n$. These estimates can be found in [12]. They imply that the number of vectors $(\mathbf{q}, \mathbf{r}, \mathbf{s})$ is bounded. Therefore, we may restrict our attention to considering polynomials $P \in P_{n}(H)$ with the same triple of vectors $(\mathbf{q}, \mathbf{r}, \mathbf{s})$ and denote this set by $P_{n}(H, \mathbf{q}, \mathbf{r}, \mathbf{s})$. The proofs of the following two lemmas can be found in [7] and [4], respectively. 
Lemma 2. Let $P \in P_{n}(H, \mathbf{q}, \mathbf{r}, \mathbf{s})$. Then

$$
\begin{aligned}
& \left|P^{(l)}\left(\alpha_{1}\right)\right|<c(n) H^{1-q_{l}+(n-l) \epsilon_{1}}, \\
& \left|P^{(l)}\left(\beta_{1}\right)\right|<c(n) H^{1-r_{l}+(n-l) \epsilon_{1}}, \\
& \left|P^{(l)}\left(\gamma_{1}\right)\right|_{p}<c(n) H^{-s_{l}+(n-l) \epsilon_{1}}, \quad 1 \leqslant l \leqslant n-1 .
\end{aligned}
$$

Lemma 3. Let $G(w)$ be the set of $\mathbf{u}(x, y, z)$ for which the inequality

$$
|P(x)||P(z)|^{2}|P(w)|_{p}<H^{-w}
$$

has infinitely many solutions $P$ with $n=\operatorname{deg} P(f) \geqslant 3$ and $H=H(P)$. Then, for $w>n-2$,

$$
\mu(G(w))=0 .
$$

Lemma 4. Let $z \in S\left(\beta_{1}\right)$. Then

$$
\left|z-\beta_{1}\right| \leqslant n|P(z)|\left|P^{\prime}(z)\right|^{-1}
$$

Proof. Write the derivative of $P$ as $P^{\prime}(z)=\left(a_{n}\left(z-\beta_{1}\right)\left(z-\beta_{2}\right) \ldots\left(z-\beta_{n}\right)\right)^{\prime}$ to obtain

$$
\left|P^{\prime}(z)\right||P(z)|^{-1}=\sum_{i=1}^{n}\left|z-\beta_{i}\right|^{-1} \leqslant n\left|z-\beta_{1}\right|^{-1} .
$$

Clearly, (13) follows.

It should also be similarly clear that inequality (13) holds for $x \in S\left(\alpha_{1}\right)$, namely,

$$
\left|x-\alpha_{1}\right| \leqslant n|P(x)|\left|P^{\prime}(x)\right|^{-1} \text {. }
$$

In the $p$-adic case for $w \in S\left(\gamma_{1}\right)$,

$$
\left|w-\gamma_{1}\right|_{p} \leqslant|P(w)|_{p}\left|P^{\prime}(w)\right|_{p}^{-1} .
$$

From the Taylor decomposition of $P^{\prime}(z)$ in the neighborhood of the root $\beta_{1}$ we obtain

$$
P^{\prime}(z)=\sum_{j=1}^{n}((j-1) !)^{-1} P^{(j)}\left(\beta_{1}\right)\left(z-\beta_{1}\right)^{j-1}
$$

The RHS of the above equation is estimated by using Lemma 1, (6), (8), (12), and (13) for sufficiently large $Q$ so that

$$
\left|P^{\prime \prime}(z)\right|\left|z-\beta_{1}\right|<c(n) Q^{1-r_{2}+(n-2) \epsilon_{1}} c_{3} Q^{-w_{2}}\left|P^{\prime}(z)\right|^{-1}<\frac{1}{2(n-1)}\left|P^{\prime}(z)\right| .
$$

From (15) and similar inequalities for the other terms in (14) we obtain that

$$
P^{\prime}(z)=P^{\prime}\left(\beta_{1}\right)+R
$$


with $|R|<\left|P^{\prime}(z)\right| / 2$. From this we have

$$
\left|P^{\prime}(z)\right| / 2<\left|P^{\prime}\left(\beta_{1}\right)\right|<3 / 2\left|P^{\prime}(z)\right| .
$$

Similar estimates are easily obtained for the real case. Then, system (8) can be rewritten as

$$
\begin{aligned}
& \frac{1}{2} Q^{1 / 2}<\left|P^{\prime}\left(\alpha_{1}\right)\right|<\frac{3}{2} c_{5} Q, \\
& \frac{1}{2} Q^{1 / 2}<\left|P^{\prime}\left(\beta_{1}\right)\right|<\frac{3}{2} c_{5} Q \\
& Q^{-\epsilon}<\left|P^{\prime}\left(\gamma_{1}\right)\right|_{p} \leqslant c_{5} .
\end{aligned}
$$

From (6), (11), and (16) it follows that all $\mathbf{u} \in S\left(\alpha_{1}\right) \times S\left(\beta_{1}\right) \times S\left(\gamma_{1}\right)$ are contained in the parallelepiped

$$
\sigma(P):=\left\{\begin{array}{l}
\left|x-\alpha_{1}\right|<c_{3} c(n) Q^{-w_{1}}\left|P^{\prime}\left(\alpha_{1}\right)\right|^{-1}, \\
\left|z-\beta_{1}\right|<c_{3} c(n) Q^{-w_{2}}\left|P^{\prime}\left(\beta_{1}\right)\right|^{-1} \\
\left|w-\gamma_{1}\right|_{p}<c_{3} c(n) Q^{-w_{3}}\left|P^{\prime}\left(\gamma_{1}\right)\right|_{p}^{-1} .
\end{array}\right.
$$

For numbers $w_{3}, w_{4}, w_{5}$ satisfying

$$
w_{4} \leqslant w_{1}, w_{5} \leqslant w_{2}, \quad w_{6}+\epsilon<w_{3}, \quad w_{4}+2 w_{5}+w_{6}=n-3, \quad w_{4}, w_{5}, w_{6} \geqslant 0,
$$

define the parallelepiped

$$
\sigma_{1}(P):=\left\{\begin{array}{l}
\left|x-\alpha_{1}\right|<c_{4}(n) Q^{-w_{1}+w_{4}}\left|P^{\prime}\left(\alpha_{1}\right)\right|^{-1} \\
\left|z-\beta_{1}\right|<c_{4} c(n) Q^{-w_{2}+w_{5}}\left|P^{\prime}\left(\beta_{1}\right)\right|^{-1} \\
\left|w-\gamma_{1}\right|_{p}<c_{4} c(n) Q^{-w_{3}+w_{6}}\left|P^{\prime}\left(\gamma_{1}\right)\right|_{p}^{-1}
\end{array}\right.
$$

and choose a constant $c_{4}$ such that $\sigma(P) \subset \sigma_{1}(P)$.

Develop the polynomial $P(t)\left(t=x, t=z, t=w\right.$ as appropriate) as a Taylor series on $\sigma_{1}(P)$ in the neighborhoods of the roots $\alpha_{1}, \beta_{1}$, and $\gamma_{1}$ and estimate from above $|P(x)|,|P(z)|$, and $|P(w)|_{p}$. For example, in the complex case (using condition (16)),

$$
\begin{aligned}
& P(z)=\sum_{i=1}^{n}(i !)^{-1} P^{(i)}\left(\beta_{1}\right)\left(z-\beta_{1}\right)^{i}, \\
& \left|P^{\prime}\left(\beta_{1}\right)\right|\left|z-\beta_{1}\right|<c_{4} c(n) Q^{-w_{2}+w_{5}}, \\
& (i !)^{-1}\left|P^{(i)}\left(\beta_{1}\right)\right|\left|z-\beta_{1}\right|^{(i)}<c_{4} c(n) Q^{1-i\left(w_{2}-w_{5}\right)-i / 2}<c_{4} c(n) Q^{-w_{2}+w_{5}}
\end{aligned}
$$

with $2 \leqslant i \leqslant n$. Hence,

$$
|P(z)|<c_{4} c(n) Q^{-w_{2}+w_{5}} .
$$

Similarly, in the real and $p$-adic cases, one can show that

$$
\begin{aligned}
& |P(x)|<c_{4} c(n) Q^{-w_{1}+w_{4}}, \\
& |P(w)|_{p}<c_{4} c(n) Q^{-w_{3}+w_{6}} .
\end{aligned}
$$

From the definitions of $\sigma(P)$ and $\sigma_{1}(P)$ it follows that

$$
\mu(\sigma(P)) / \mu\left(\sigma_{1}(P)\right)<c_{4}^{-4} c(n) Q^{-\left(w_{4}+2 w_{5}+w_{6}\right)}=c_{4}^{-4} c(n) Q^{-n+3} .
$$


Fix the integer vector $\mathbf{b}=\left(a_{4}, a_{5}, \ldots, a_{n}\right)$ of the coefficients of $P \in P_{n}(H, \mathbf{q}, \mathbf{r}, \mathbf{s})$ and denote the subset of polynomials with the same vector $\mathbf{b}$ by $P_{n, \mathbf{b}}(H, \mathbf{q}, \mathbf{r}, \mathbf{s})$. Now, consider the relative position of the parallelepipeds $\sigma_{1}(P)$ for different polynomials $P_{1}$ and $P_{2}$ in $P_{n, \mathbf{b}}(H, \mathbf{q}, \mathbf{r}, \mathbf{s})$. The parallelepiped $\sigma_{1}\left(P_{1}\right)$ is called essential if there is a polynomial $P_{2} \in P_{n, \mathbf{b}}(H, \mathbf{q}, \mathbf{r}, \mathbf{s})$ such that

$$
\mu\left(\sigma_{1}\left(P_{1}\right) \cap \sigma_{1}\left(P_{2}\right)\right)<1 / 2 \mu\left(\sigma_{1}\left(P_{1}\right)\right)
$$

and inessential otherwise. Hence, $P_{1}$ is inessential if there exists $P_{2} \in P_{n, \mathbf{b}}(H, \mathbf{q}, \mathbf{r}, \mathbf{s})$ such that

$$
\mu\left(\sigma_{1}\left(P_{1}\right) \cap \sigma_{1}\left(P_{2}\right)\right) \geqslant 1 / 2 \mu\left(\sigma_{1}\left(P_{1}\right)\right) .
$$

Let the parallelepiped $\sigma_{1}\left(P_{1}\right)$ be essential. Then from (20) it follows that

$$
\sum_{P_{1} \in P_{n, \mathbf{b}}(H, \mathbf{q}, \mathbf{r}, \mathbf{s})} \mu\left(\sigma_{1}\left(P_{1}\right)\right) \ll \mu\left(T_{0}\right)
$$

By (19) and (21) we obtain

$$
\sum_{P_{1} \in P_{n, \mathbf{b}}(H, \mathbf{q}, \mathbf{r}, \mathbf{s})} \mu\left(\sigma\left(P_{1}\right)\right) \leqslant c_{4}^{-4} c(n) \mu\left(T_{0}\right) Q^{-\left(w_{4}+2 w_{5}+w_{6}\right)} \leqslant c_{4}^{-4} c(n) Q^{-n+3} \mu\left(T_{0}\right) .
$$

Since the number of vectors $\mathbf{b}$ is $\ll Q^{n-4}$, from (22) we have

$$
\sum_{\left|a_{j}\right| \leqslant Q} \sum_{\mathbf{b}} \sum_{P_{1} \in P_{n, \mathbf{b}}(H, \mathbf{q}, \mathbf{r}, \mathbf{s})} \mu\left(\sigma\left(P_{1}\right)\right)<c_{4}^{-4} c(n) Q^{1+n-4-(n-3)} \mu\left(T_{0}\right)<c_{4}^{-4} c(n) \mu\left(T_{0}\right) .
$$

Now let the parallelepiped $\sigma_{1}\left(P_{1}\right)$ be inessential, and define $\sigma_{1}\left(P_{1}, P_{2}\right)=\sigma_{1}\left(P_{1}\right) \cap \sigma_{1}\left(P_{2}\right)$. Inequalities (17))-(18) hold simultaneously on $\sigma_{1}\left(P_{1}, P_{2}\right)$ for $P_{1}$ and $P_{2}$. By (17)-(18) the polynomial $R=P_{2}-P_{1}$ with $\operatorname{deg} R \leqslant 3$ and $H(R) \leqslant 2 H$ satisfies

$$
\begin{aligned}
& |R(x)|<c_{4} c(n) Q^{-w_{1}+w_{4}}, \\
& |R(z)|<c_{4} c(n) Q^{-w_{2}+w_{5}} \\
& |R(w)|_{p}<c_{4} c(n) Q^{-w_{3}+w_{6}} .
\end{aligned}
$$

Using the estimates for $P^{\prime}(t)$ in (16), it is readily verified that, on $\sigma_{1}\left(P_{1}, P_{2}\right)$ and for $Q$ sufficiently large,

$$
\begin{aligned}
& P^{\prime}(x)=\sum_{i=1}^{n}((i-1) !)^{-1} P^{(i)}\left(\alpha_{1}\right)\left(x-\alpha_{1}\right)^{i-1}, \\
& \left|P^{\prime}\left(\alpha_{1}\right)\right|<\frac{3}{2} c_{5} Q, \\
& \left|P^{(i)}\left(\alpha_{1}\right)\right|\left|x-\alpha_{1}\right|^{(i-1)}<c_{4}^{i-1} c(n) Q^{1-(i-1)\left(w_{1}-w_{4}\right)}\left|P^{\prime}\left(\alpha_{1}\right)\right|^{-(i-1)}<c_{4} c_{5} c(n) Q, \quad 3 \leqslant i \leqslant n .
\end{aligned}
$$

It follows that $\left|P^{\prime}(x)\right|<c_{4} c_{5} c(n) Q$ and

$$
\left|R^{\prime}(x)\right|<c_{4} c_{5} c(n) Q
$$

Similarly, inequalities in the complex and $p$-adic cases can be obtained so that

$$
\begin{aligned}
& \left|R^{\prime}(z)\right|<c_{4} c_{5} c(n) Q, \\
& \left|R^{\prime}(w)\right|_{p}<c_{4} c_{5} c(n) .
\end{aligned}
$$


We denote by $\alpha_{i}(R), \beta_{i}(R)$, and $\gamma_{i}(R)$ the roots $R$ in $\mathbb{R}, \mathbb{C}$, and $\mathbb{Q}_{p}$. From (24) and (3) it follows that the roots $\alpha_{1}(R), \beta_{1}(R)$, and $\bar{\beta}_{1}(R)$ are at least $\delta_{1}$ apart. Thus,

$$
H(R)<c\left(n, \delta_{1}\right)\left|R^{\prime}(x)\right|<c_{4} c_{5} c\left(n, \delta_{1}\right) Q .
$$

We estimate the measure of $\mathbf{u}$ for which the system of inequalities (24) and condition (25) holds. Whence,

$$
\begin{aligned}
& \left|x-\alpha_{1}(R)\right|<c_{4} c(n) Q^{-w_{1}+w_{4}}|H(R)|^{-1}, \\
& \left|z-\beta_{1}(R)\right|<c_{4} c(n) Q^{-w_{2}+w_{5}}|H(R)|^{-1} \\
& \left|w-\gamma_{1}(R)\right|_{p}<c_{4} c(n) Q^{-w_{3}+w_{6}}|H(R)|_{p}^{-1} .
\end{aligned}
$$

Using this gives an estimate for the measure of $\mathbf{u}$ in the inessential parallelepipeds, namely,

$$
c_{4}^{4} c(n) Q^{-\left(w_{1}+2 w_{2}+w_{3}\right)+\left(w_{4}+2 w_{5}+w_{6}\right)} H(R)^{-4}=c_{4}^{4} c(n) Q^{-1} H(R)^{-3} .
$$

Summing the previous estimate over the remaining coefficients shows that none of these sums exceeds $H(R) \times(|I|, \mu(K), \mu(D))$. This further implies that the measure of the union of all inessential parallelepipeds is

$$
\ll c_{4}^{4} Q^{-1} H(R) \mu\left(T_{0}\right) \ll c_{4}^{4} c_{5} \mu\left(T_{0}\right)
$$

by (25). This completes the proof.

There are six other cases where one or more of the inequalities in (7) does not hold.

1. Assume that the inequality $\left|P^{\prime}(z)\right|>c_{6} Q$ holds for some constant $c_{6}>0$. In this case, one must show that the set $B_{12}$ of solutions of system (6) and the system

$$
Q^{1 / 2}<\left|P^{\prime}(x)\right|<c_{8} Q, \quad\left|P^{\prime}(z)\right|>c_{6} Q, \quad Q^{-1 / 2}<\left|P^{\prime}(w)\right|_{p}<c_{7}
$$

for some $c_{7}$ has the measure $\mu\left(B_{12}\right)<2^{-6} \mu\left(T_{0}\right)$.

2. Assume that the inequality $\left|P^{\prime}(x)\right|>c_{8} Q$ holds for some constant $c_{8}>0$. In this case, one must show that the set $B_{13}$ of solutions of system (6) and the system

$$
\left|P^{\prime}(x)\right|>c_{8} Q, \quad Q^{1 / 2}<\left|P^{\prime}(z)\right|<c_{9} Q, \quad Q^{-1 / 2}<\left|P^{\prime}(w)\right|_{p}<c_{9}
$$

for some constant $c_{9}<c_{9}^{\prime}$ has the measure $\mu\left(B_{13}\right)<2^{-6} \mu\left(T_{0}\right)$.

3. Assume that the inequality $\left|P^{\prime}(w)\right|_{p}>c_{10}$ holds for some constant $c_{10}>0$. Then one must show that the set $B_{14}$ of solutions of system (6) and the system

$$
Q^{1 / 2}<\left|P^{\prime}(x)\right|<c_{11} Q, \quad Q^{1 / 2}<\left|P^{\prime}(z)\right|<c_{11} Q, \quad\left|P^{\prime}(w)\right|_{p}>c_{10}
$$

for some $c_{11}<c_{11}^{\prime}$ has the measure $\mu\left(B_{14}\right)<2^{-6} \mu\left(T_{0}\right)$.

4. Assume that the inequalities $\left|P^{\prime}(x)\right|>c_{12} Q$ and $\left|P^{\prime}(z)\right|>c_{12} Q$ hold for some constant $c_{12}$. Then one must show that the set $B_{15}$ of solutions of system (6) and the system

$$
\left|P^{\prime}(x)\right|>c_{12} Q, \quad\left|P^{\prime}(z)\right|>c_{12} Q, \quad Q^{-1 / 2}<\left|P^{\prime}(w)\right|_{p}<c_{13}
$$

for some $c_{13}<c_{14}^{\prime}$ has the measure $\mu\left(B_{15}\right)<2^{-6} \mu\left(T_{0}\right)$. 
5. Assume that the inequalities $\left|P^{\prime}(x)\right|>c_{14} Q$ and $\left|P^{\prime}(w)\right|_{p}>c_{14}$ hold for some constant $c_{14}$. Then one must show that the set $B_{16}$ of solutions of system (6) and the system

$$
\left|P^{\prime}(x)\right|>c_{14} Q, \quad Q^{1 / 2}<\left|P^{\prime}(z)\right|<c_{15} Q, \quad\left|P^{\prime}(w)\right|_{p}>c_{14}
$$

for some constant $c_{15}<c_{15}^{\prime}$ has the measure $\mu\left(B_{16}\right)<2^{-6} \mu\left(T_{0}\right)$.

6. Assume that the inequalities $\left|P^{\prime}(z)\right|>c_{16} Q$ and $\left|P^{\prime}(w)\right|_{p}>c_{16}$ hold for some constant $c_{16}$. Then one must show that the set $B_{17}$ of solutions of system (6) and the system

$$
Q^{1 / 2}<\left|P^{\prime}(x)\right|<c_{17} Q, \quad\left|P^{\prime}(z)\right|>c_{16} Q, \quad\left|P^{\prime}(w)\right|_{p}>c_{16}
$$

for some constant $c_{17}<c_{17}^{\prime}$ has the measure $\mu\left(B_{17}\right)<2^{-6} \mu\left(T_{0}\right)$.

The proofs of these six cases are based on the simple statement that, according to Lemma 1 , if we have $\left|P^{\prime}(x)\right|>c(n) Q$ for all $x \in I$, then the measure of such $x$ which satisfy the inequality $|P(x)|<c_{3} Q^{-w_{1}}$ is at most $c(n) Q^{-w_{1}-1}$. Similar results hold for the measure of a disk in $\mathbb{C}$ in which $|P(z)|<c_{3} Q^{-w_{2}}$ and $\left|P^{\prime}(z)\right|>c(n) Q$ and for the measure of a cylinder in $\mathbb{Q}_{p}$ in which $|P(w)|_{p}<c_{3} Q^{-w_{3}}$ and $\left|P^{\prime}(w)\right|>$ $c(n)$.

As before, we will construct similar sets to $\sigma(P)$ and $\sigma_{1}(P)$ for some of the above listed cases.

For Case 1, we obtain

$$
\begin{gathered}
\sigma(P):=\left\{\begin{array}{l}
\left|x-\alpha_{1}\right|<c_{18} Q^{-w_{1}}\left|P^{\prime}\left(\alpha_{1}\right)\right|^{-1} \\
\left|z-\beta_{1}\right|<c_{18} Q^{-w_{2}-1} \\
\left|w-\gamma_{1}\right|_{p}<c_{18} Q^{-w_{3}}\left|P^{\prime}\left(\gamma_{1}\right)\right|_{p}^{-1}
\end{array}\right. \\
\sigma_{1}(P):=\left\{\begin{array}{l}
\left|x-\alpha_{1}\right|<c_{19} Q^{-w_{1}+w_{4}}\left|P^{\prime}\left(\alpha_{1}\right)\right|^{-1} \\
\left|z-\beta_{1}\right|<c_{19} Q^{-w_{2}+w_{5}-1} \\
\left|w-\gamma_{1}\right|_{p}<c_{19} Q^{-w_{3}+w_{6}}\left|P^{\prime}\left(\gamma_{1}\right)\right|_{p}^{-1}
\end{array}\right.
\end{gathered}
$$

For Case 2,

$$
\begin{gathered}
\sigma(P):=\left\{\begin{array}{l}
\left|x-\alpha_{1}\right|<c_{20} Q^{-w_{1}-1} \\
\left|z-\beta_{1}\right|<c_{20} Q^{-w_{2}}\left|P^{\prime}\left(\beta_{1}\right)\right|^{-1} \\
\left|w-\gamma_{1}\right| c_{20} Q^{-w_{3}}\left|P^{\prime}\left(\gamma_{1}\right)\right|_{p}^{-1}
\end{array}\right. \\
\sigma_{1}(P):=\left\{\begin{array}{l}
\left|x-\alpha_{1}\right|<c_{21} Q^{-w_{1}+w_{4}-1} \\
\left|z-\beta_{1}\right|<c_{21} Q^{-w_{2}+w_{5}}\left|P^{\prime}\left(\beta_{1}\right)\right|^{-1} \\
\left|w-\gamma_{1}\right|_{p}<c_{21} Q^{-w_{3}+w_{6}}\left|P^{\prime}\left(\gamma_{1}\right)\right|_{p}^{-1}
\end{array}\right.
\end{gathered}
$$

For Case 6,

$$
\begin{aligned}
& \sigma(P):=\left\{\begin{array}{l}
\left|x-\alpha_{1}\right|<c_{22} Q^{-w_{1}}\left|P^{\prime}\left(\alpha_{1}\right)\right|^{-1} \\
\left|z-\beta_{1}\right|<c_{22} Q^{-w_{2}-1} \\
\left|w-\gamma_{1}\right|_{p}<c_{22} Q^{-w_{3}}
\end{array}\right. \\
& \sigma_{1}(P):=\left\{\begin{array}{l}
\left|x-\alpha_{1}\right|<c_{23} Q^{-w_{1}+w_{4}}\left|P^{\prime}\left(\alpha_{1}\right)\right|^{-1}, \\
\left|z-\beta_{1}\right|<c_{23} Q^{-w_{2}+w_{5}-1} \\
\left|w-\gamma_{1}\right|_{p}<c_{23} Q^{-w_{3}+w_{6}}
\end{array}\right.
\end{aligned}
$$


In every case, the polynomials $P$ and $P^{\prime}$ are developed as Taylor series on the parallelepipeds $\sigma_{1}(P)$, and an upper bound is obtained. Then, a vector $\mathbf{b}=\left(a_{4}, a_{5}, \ldots, a_{n}\right)$ is fixed, and the essential and inessential parallelepipeds are investigated. Similar results to (23) and (26) are readily obtained.

The constant $c_{4}$ in (23) is chosen so that the estimate is at most $2^{-7} \mu\left(T_{0}\right)$. Then, in (26), the constant $c_{5}$ is chosen small enough so that this estimate also is at most $2^{-7} \mu\left(T_{0}\right)$. Thus, in all seven cases, the estimate of the measure of $\mathbf{u}$ for which the systems of inequalities (6) and (8) hold is at most $\frac{7}{32} \mu\left(T_{0}\right)<\frac{1}{4} \mu\left(T_{0}\right)$.

If in (8) one of the derivatives is less than $Q^{1 / 2}$ in the real or complex case, or $\left|P^{\prime}(w)\right|_{p}<Q^{-\epsilon}$ in the $p$-adic case, then, as is shown in [8] and [9], the measure of $\mathbf{u}$ tends to zero as $Q \rightarrow \infty$. Hence, there exists a sufficiently large number $Q>Q_{0}$ such that the measure of $\mathbf{u}$ is $<\frac{1}{4} \mu\left(T_{0}\right)$.

Thus, the systems of inequalities (6) and (7) hold on some set $B_{2}$ with $\mu\left(B_{2}\right)>\mu\left(T_{0}\right) / 2$.

Now we use Lagrange's Theorem and Hensel's lemma (stated below).

Lemma 5 [Hensel]. Let $P \in \mathbb{Z}_{p}[x], \xi=\xi_{0} \in \mathbb{Z}_{p}$, and $|P(\xi)|_{p}<\left|P^{\prime}(\xi)\right|_{p}^{2}$. Then, as $n \rightarrow \infty$, the sequence $\xi_{n+1}=\xi_{n}-P\left(\xi_{n}\right) P^{\prime}\left(\xi_{n}\right)^{-1}$ tends to some root $\alpha \in \mathbb{Z}_{p}$ of $P$ and $|\alpha-\xi|_{p}<|P(\xi)|_{p}\left|P^{\prime}(\xi)\right|_{p}^{-2}<1$.

The construction of an optimal regular system will now be demonstrated. From the first inequalities of systems (6) and (7) we obtain that there exists a real root $\alpha_{1}$ of $P$ such that

$$
\left|x-\alpha_{1}\right|<c(n) Q^{-w_{1}-1}
$$

from the second inequalities it also follows that

$$
\left|z-\beta_{1}\right|<c(n) Q^{-w_{2}-1}
$$

finally, from the third inequalities (and using Hensel's lemma) we get that if $|P(w)|_{p}<c_{24}\left|P^{\prime}(w)\right|_{p}^{2}$, then there exists a $p$-adic root $\gamma_{1} \in \mathbb{Q}_{p}$ such that

$$
\left|w-\gamma_{1}\right|_{p}<c_{24} Q^{-w_{3}}
$$

The union $B$ of parallelepipeds defined by (27))-(29) has the measure

$$
\mu(B)>\mu\left(T_{0}\right) / 2,
$$

as proved above.

Now we choose a maximal system of vectors $\boldsymbol{\nu}_{1}, \boldsymbol{\nu}_{2}, \ldots, \boldsymbol{\nu}_{t} \in T_{0}$, with $\boldsymbol{\nu}_{j}=\left(\alpha_{j}, \beta_{j}, \gamma_{j}\right), 1 \leqslant j \leqslant t$, $\alpha_{j} \in \mathbb{R}, \beta_{j} \in \mathbb{C}, \operatorname{Im} \beta_{j}>\delta_{1}, \gamma_{j} \in \mathbb{Q}_{p}$, which satisfy the condition

$$
H\left(\boldsymbol{\nu}_{j}\right) \leqslant c(n) Q
$$

also, for all $i, j, 1 \leqslant i<j \leqslant t$, the parallelepipeds

$$
T_{i}=\left\{\mathbf{u} \in T_{0}:\left|x-\alpha_{i}\right|<c_{25} Q^{-w_{1}-1},\left|z-\beta_{i}\right|<c_{25} Q^{-w_{2}-1},\left|w-\gamma_{i}\right|_{p}<c_{25} Q^{-w_{3}}\right\}
$$

do not intersect.

For the proof of the regularity of this system, it is necessary to obtain the estimate

$$
t>c(n) Q^{n+1} \mu\left(T_{0}\right) .
$$

In order to do this, we take all vectors $\nu_{j_{i}}, 1 \leqslant i \leqslant t_{1}$, satisfying (31), so that all the parallelepipeds $T_{j_{i}}$ constructed on the vectors $\boldsymbol{\nu}_{j_{i}}$ intersect with the parallelepipeds $T_{i}$ which are constructed on the vectors 
$\nu_{i}, 1 \leqslant i \leqslant t$, and

$$
\mu\left(\bigcup_{i=1}^{t} T_{i}\right) \geqslant \frac{1}{8} \mu\left(\bigcup_{i=1}^{t_{1}} T_{j_{i}}\right) .
$$

By (30), the RHS of (33) is $>2^{-4} \mu\left(T_{0}\right)$, and the LHS (the left-hand side) of (33) is at most $t c_{25} Q^{-n-1}$, whence inequality (32) follows.

Proof of Theorem 2. For any vector $\boldsymbol{\nu}=(\alpha, \beta, \gamma)$, define the parallelepiped

$$
\sigma(\boldsymbol{\nu})=\left\{\mathbf{u} \in \mathbb{R} \times \mathbb{C} \times \mathbb{Q}_{p}: \begin{array}{l}
|x-\alpha|<H(\boldsymbol{\nu})^{-v_{1}-1} \Psi^{\lambda_{1}}(H), \\
|z-\beta|<H(\boldsymbol{\nu})^{-v_{2}-1} \Psi^{\lambda_{2}}(H), \\
|w-\gamma|_{p}<H(\boldsymbol{\nu})^{-v_{3}} \Psi^{\lambda_{3}}(H) .
\end{array}\right\}
$$

Clearly, the set of solutions of (2) contains all $\mathbf{u}$ belonging to infinitely many parallelepipeds $\sigma(\boldsymbol{\nu})$.

The following two lemmas are proved in [1] and [13, Chapter 2], respectively.

Lemma 6. Let $A \subset T$ be a measurable set. If there exists a positive constant $c_{1}<1$ such that $\mu\left(A \cap T^{\prime}\right)>$ $c_{1} \mu\left(T^{\prime}\right)$ for any parallelepiped $T^{\prime} \subset T$, then $A$ has full measure, i.e., $\mu(A)=\mu(T)$.

Lemma 7. Let $E_{i} \subset T$ be a sequence of measurable sets, and let $E$ be the set of points belonging to infinitely many of the $E_{i}$. If each $E_{i}$ is totally bounded and $\sum_{i=1}^{\infty} \mu\left(E_{i}\right)=\infty$, then

$$
\mu(E) \geqslant \limsup _{N \rightarrow \infty} \frac{\left(\sum_{i=1}^{N} \mu\left(E_{i}\right)\right)^{2}}{\sum_{i=1}^{N} \sum_{j=1}^{N} \mu\left(E_{i} \bigcap E_{j}\right)} .
$$

The next two lemmas are from [1].

Lemma 8. Let $\left\{a_{i}\right\}_{i=1}^{\infty}$ be a decreasing sequence of positive numbers such that $\sum_{i=1}^{\infty} a_{i}=\infty$. Define $b_{i}=$ $\min \left\{a_{i} ; i^{-1}\right\}$. Then $\left\{b_{i}\right\}_{i=1}^{\infty}$ also decreases, and $\sum_{i=1}^{\infty} b_{i}=\infty$.

Lemma 9. Given a decreasing sequence $\Psi(h)$ of positive numbers such that $\sum_{h=1}^{\infty} \Psi(h)$ converges/diverges, for any number $c>0$, the series $\sum_{k=0}^{\infty} 2^{k} \Psi\left(c 2^{k}\right)$ converges/diverges.

Clearly, from these we may assume without loss of generality that

$$
\Psi(h)<h^{-1} / 2 \text { for all } h \in \mathbb{N} .
$$

Define $\Phi(h)=h \Psi(h)$. By Lemma 9,

$$
\sum_{k=0}^{\infty} \Phi\left(2^{k}\right)=\infty
$$

For each $Q$, we can find $k \in \mathbb{N}$ such that $2^{k} \leqslant Q<2^{k+1}$. All the inequalities used in the construction of the regular system are now rewritten in terms of $k$. Hence, there exists a positive constant $k_{0}=k_{0}\left(n, T_{0}\right)$ such that, for any $k \geqslant k_{0}$, there exists a set of vectors

$$
A_{k}\left(T_{0}\right)=\left\{\boldsymbol{\nu}_{1}, \ldots, \boldsymbol{\nu}_{t_{k}}\right\}
$$

such that

$$
H\left(\boldsymbol{\nu}_{i}\right) \leqslant 2^{k}
$$


for all $\boldsymbol{\nu}_{i} \in A_{k}\left(T_{0}\right)$. The parallelepipeds

$$
\Pi\left(\boldsymbol{\nu}_{i}\right):=\left\{\mathbf{u} \in T: \begin{array}{l}
\left|x-\alpha_{i}\right| \leqslant 2^{-\left(w_{1}+1\right) k}, \\
\left|z-\beta_{i}\right| \leqslant 2^{-\left(w_{2}+1\right) k} \\
\left|w-\gamma_{i}\right|_{p} \leqslant 2^{-w_{3} k},
\end{array}\right\}
$$

do not intersect for any $\boldsymbol{\nu}_{i} \neq \boldsymbol{\nu}_{j}$, so

$$
c_{25} 2^{(n+1) k} \mu\left(T_{0}\right) \leqslant t_{k} \leqslant c_{26} 2^{(n+1) k} \mu\left(T_{0}\right) .
$$

Define the parallelepipeds

$$
E_{k}(\boldsymbol{\nu}):=\left\{\begin{array}{ll}
\mathbf{u} \in T: & \left|x-\alpha_{1}\right| \leqslant 2^{-\left(v_{1}+1\right) k} \Psi^{\lambda_{1}}\left(2^{k}\right), \\
& \left|z-\beta_{1}\right| \leqslant 2^{-\left(v_{2}+1\right) k} \Psi^{\lambda_{2}}\left(2^{k}\right), \\
\left|w-\gamma_{1}\right| & \leqslant 2^{-v_{3} k} \Psi^{\lambda_{3}}\left(2^{k}\right),
\end{array}\right\}
$$

and let

$$
E_{k}=\bigcup_{\boldsymbol{\nu} \in A_{k}\left(T_{0}\right)} E_{k}(\boldsymbol{\nu})
$$

By (38),

$$
\mu\left(E_{k}(\boldsymbol{\nu})\right)=2 \cdot 2^{-k n} \Psi\left(2^{k}\right)=2 \cdot 2^{-k(n+1)} \Phi\left(2^{k}\right) .
$$

Clearly, $\mu\left(\mathcal{L}_{\mathbf{v}, \boldsymbol{\lambda}}(\Psi) \bigcap T_{0}\right) \geqslant \mu\left(E\left(T_{0}\right)\right)$, where $E\left(T_{0}\right)=\bigcap_{N=k_{0}}^{\infty} \bigcup_{k=N}^{\infty} E_{k}$. From (34) and (36) it follows that $E_{k}\left(\boldsymbol{\nu}_{1}\right) \cap E_{k}\left(\boldsymbol{\nu}_{2}\right)=0$ for all $\boldsymbol{\nu}_{1}, \boldsymbol{\nu}_{2} \in A_{k}\left(T_{0}\right), \boldsymbol{\nu}_{1} \neq \boldsymbol{\nu}_{2}$. Then $\mu\left(E_{k}\right)=t_{k} E_{k}(\boldsymbol{\nu})$, where $\nu \in A_{k}\left(T_{0}\right)$. By (37) and (38), we obtain

$$
2 c_{25} \Phi\left(2^{k}\right) \mu\left(T_{0}\right) \leqslant \mu\left(E_{k}\right) \leqslant 2 c_{26} \Phi\left(2^{k}\right) \mu\left(T_{0}\right) .
$$

Hence,

$$
\sum_{k=k_{0}}^{N} \mu\left(E_{k}\right) \geqslant 2 c_{25} \mu\left(T_{0}\right) \sum_{k=k_{0}}^{N} \Phi\left(2^{k}\right) .
$$

Using this and (35) gives that $\sum_{k=k_{0}}^{N} \mu\left(E_{k}\right)=\infty$.

Furthermore, choose a number $N_{0}>k_{0}$ such that

$$
\sum_{k=k_{0}}^{N_{0}} \Phi\left(2^{k}\right)>1
$$

Fix $l$ and $k$ such that $k_{0} \leqslant k<l \leqslant N$ and $N>N_{0}$. For any $\boldsymbol{\nu} \in A_{k}\left(T_{0}\right)$,

$$
E_{l} \cap E_{k}(\boldsymbol{\nu})=\bigcup_{\boldsymbol{\nu} \in A_{l}\left(T_{0}\right)} E_{l}\left(\boldsymbol{\nu}_{1}\right) \cap E_{k}\left(\boldsymbol{\nu}_{2}\right)
$$

For $\left(v_{1}, v_{2}, v_{3}\right)$ and $\left(\lambda_{1}, \lambda_{2}, \lambda_{3}\right)$ defined in Theorem 2, we shall construct a regular system with $w_{1}=$ $v_{1}+\lambda_{1}, w_{2}=v_{2}+\lambda_{2}, w_{3}=v_{3}+\lambda_{3}$. Given $\nu_{1} \in A_{k}\left(T_{0}\right)$, the number of different $\boldsymbol{\nu}_{2} \in A_{l}\left(T_{0}\right)$ satisfying the condition $E_{l}\left(\boldsymbol{\nu}_{2}\right) \bigcap E_{k}\left(\boldsymbol{\nu}_{1}\right) \neq \emptyset$ is at most

$$
\left(2+2^{-k\left(v_{1}+1\right)} \Psi^{\lambda_{1}}\left(2^{k}\right) 2^{\left(w_{1}+1\right) l}\right)\left(2+2^{-k\left(v_{2}+1\right)} \Psi^{\lambda_{2}}\left(2^{k}\right) 2^{\left(w_{2}+1\right) l}\right)^{2}\left(2+2^{-k v_{3}} \Psi^{\lambda_{3}}\left(2^{k}\right) 2^{w_{3} l}\right) .
$$


By Theorem 2, we have $v_{1}=v_{2}=(n-4) / 4, v_{3}=n / 4$. Then $w_{1}=w_{2}=(n-3) / 4, w_{3}=(n+1) / 4$, and expression (43) is a product of four identical factors. There are two cases that must be considered. Firstly, the second term in the summand is greater than the first term in every bracket in (43). Secondly, the second term in the summand does not surpass the first term in every bracket in (43).

Consider the first case. Firstly, the estimate of (43) is

$$
\leqslant 16 \cdot 2^{-k\left(v_{1}+2 v_{2}+v_{3}+3\right)} \Psi^{\lambda_{1}+2 \lambda_{2}+\lambda_{3}}\left(2^{k}\right) 2^{(n+1) l}=16 \cdot 2^{-k n} \Psi\left(2^{k}\right) 2^{(n+1) l}=16 \cdot 2^{(n+1)(l-k)} \Phi\left(2^{k}\right) .
$$

From this, (39), and (42) we obtain

$$
\begin{aligned}
\mu\left(E_{l} \cap E_{k}\left(\boldsymbol{\nu}_{1}\right)\right) & \leqslant 16 \mu E_{l}\left(\boldsymbol{\nu}_{2}\right) 2^{(n+1)(l-k)} \Phi\left(2^{k}\right) \leqslant 32 \cdot 2^{-l(n+1)+(n+1)(l-k)} \Phi\left(2^{l}\right) \Phi\left(2^{k}\right) \\
& =32 \cdot 2^{-(n+1) k} \Phi\left(2^{l}\right) \Phi\left(2^{k}\right) .
\end{aligned}
$$

Further, using (37), we have

$$
\begin{aligned}
\mu\left(E_{l} \cap E_{k}\right) & \leqslant t_{k}\left(\mu\left(E_{l} \cap E_{k}\left(\boldsymbol{\nu}_{1}\right)\right) \leqslant 32 c_{26} 2^{(n+1) k} 2^{-(n+1) k} \Phi\left(2^{l}\right) \Phi\left(2^{k}\right) \mu\left(T_{0}\right)\right. \\
& =32 c_{26} \Phi\left(2^{l}\right) \Phi\left(2^{k}\right) \mu\left(T_{0}\right) .
\end{aligned}
$$

Since $E_{k} \cap E_{l}=E_{l} \cap E_{k}$,

$$
\sum_{l=k_{0}}^{N} \sum_{k=k_{0}}^{N} \mu\left(E_{l} \cap E_{k}\right)=\sum_{k=k_{0}}^{N} \mu\left(E_{k}\right)+2 \sum_{l=k_{0}+1}^{N} \sum_{k=k_{0}}^{l-1} \mu\left(E_{l} \cap E_{k}\right) .
$$

From (40) it follows that

$$
\sum_{k=k_{0}}^{N} \mu\left(E_{k}\right) \leqslant 2 c_{26} \mu\left(T_{0}\right) \sum_{k=k_{0}}^{N} \Phi\left(2^{k}\right)
$$

Using (44), the second summand in (46) can be estimated so that

$$
2 \sum_{l=k_{0}+1}^{N} \sum_{k=k_{0}}^{l-1} \mu\left(E_{l} \cap E_{k}\right) \leqslant 64 c_{26} \mu\left(T_{0}\right) \sum_{l=k_{0}+1}^{N} \sum_{k=k_{0}}^{l-1} \Phi\left(2^{l}\right) \Phi\left(2^{k}\right) \leqslant 32 c_{26} \mu\left(T_{0}\right)\left(\sum_{k=k_{0}}^{N} \Phi\left(2^{k}\right)\right)^{2} .
$$

By (41) and (46)-(48) we obtain that

$$
\sum_{l=k_{0}}^{N} \sum_{k=k_{0}}^{N} \mu\left(E_{l} \cap E_{k}\right) \leqslant 34 c_{26} \mu\left(T_{0}\right)\left(\sum_{k=k_{0}}^{N} \Phi\left(2^{k}\right)\right)^{2} .
$$

According to the last estimate and Lemma 7,

$$
\frac{\left(\sum_{k=k_{0}}^{N} \mu\left(E_{k}\right)\right)^{2}}{\sum_{l=k_{0}}^{N} \sum_{k=k_{0}}^{N} \mu\left(E_{l} \cap E_{k}\right)} \geqslant \frac{\left(2 c_{25} \mu\left(T_{0}\right)\right)^{2}\left(\sum_{k=k_{0}}^{N} \Phi\left(2^{k}\right)\right)^{2}}{34 c_{26} \mu\left(T_{0}\right)\left(\sum_{k=k_{0}}^{N} \Phi\left(2^{k}\right)\right)^{2}}=\frac{2}{17} c_{25}^{2} c_{26}^{-1} \mu\left(T_{0}\right)=c_{27} \mu\left(T_{0}\right)
$$

for any $N>N_{0}$. From Lemma 7 it therefore follows that $\mu\left(E\left(T_{0}\right)\right)>c_{27} \mu\left(T_{0}\right)$ and $\mu\left(\mathcal{L}_{\mathbf{v}, \boldsymbol{\lambda}}(\Psi) \cap T_{0}\right) \geqslant$ $c_{27} \mu\left(T_{0}\right)$ for any finite parallelepiped $T_{0}$. The proof of Theorem 2 can be completed by using Lemma 6.

Consider the second case. This time, the estimate for (43) is $\leqslant 256$. Using this, (39), and (42) gives

$$
\mu\left(E_{l} \cap E_{k}\left(\boldsymbol{\nu}_{1}\right)\right) \leqslant 256 \mu\left(E_{l}\left(\boldsymbol{\nu}_{2}\right)\right)=2^{9} \cdot 2^{-l(n+1)} \Phi\left(2^{l}\right)
$$


and therefore, by (37),

$$
\mu\left(E_{l} \cap E_{k}\right) \leqslant 2^{9} c_{26} \mu\left(T_{0}\right) 2^{-(l-k)(n+1)} \Phi\left(2^{l}\right) .
$$

Since $E_{k} \cap E_{l}=E_{l} \cap E_{k}$, we have (46). The double sum on the RHS of (46) is estimated by means of (49) to obtain

$$
\begin{aligned}
2 \sum_{l=k_{0}+1}^{N} \sum_{k=k_{0}}^{l-1} \mu\left(E_{l} \cap E_{k}\right) & \leqslant 2^{10} c_{26} \mu\left(T_{0}\right) \sum_{l=k_{0}+1}^{N} \sum_{k=k_{0}}^{l-1} 2^{-(l-k)(n+1)} \Phi\left(2^{l}\right) \\
& \leqslant 2^{10-n} c_{26} \mu\left(T_{0}\right) \sum_{l=k_{0}+1}^{N} \Phi\left(2^{l}\right) \\
& \leqslant 2^{7} c_{26} \mu\left(T_{0}\right) \sum_{l=k_{0}+1}^{N} \Phi\left(2^{l}\right) .
\end{aligned}
$$

Thus, from (40), (41), and (50) we conclude that

$$
\sum_{l=k_{0}}^{N} \sum_{k=k_{0}}^{N} \mu\left(E_{l} \cap E_{k}\right) \leqslant 130 c_{26} \mu\left(T_{0}\right)\left(\sum_{k=k_{0}}^{N} \Phi\left(2^{k}\right)\right)^{2}
$$

This and (40) imply

$$
\frac{\left(\sum_{k=k_{0}}^{N} \mu\left(E_{k}\right)\right)^{2}}{\sum_{l=k_{0}}^{N} \sum_{k=k_{0}}^{N} \mu\left(E_{l} \cap E_{k}\right)} \geqslant \frac{\left(2 c_{25} \mu\left(T_{0}\right)\right)^{2}\left(\sum_{k=k_{0}}^{N} \Phi\left(2^{k}\right)\right)^{2}}{130 c_{26} \mu\left(T_{0}\right)\left(\sum_{k=k_{0}}^{N} \Phi\left(2^{k}\right)\right)^{2}}=\frac{2}{65} c_{25}^{2} c_{26}^{-1} \mu\left(T_{0}\right)=c_{28} \mu\left(T_{0}\right)
$$

for any $N>N_{0}$. It follows that $\mu\left(E\left(T_{0}\right)\right)>c_{28} \mu\left(T_{0}\right)$ and from Lemma 7 that $\mu\left(\mathcal{L}_{\mathbf{v}, \boldsymbol{\lambda}}(\Psi) \cap T_{0}\right) \geqslant$ $c_{28} \mu\left(T_{0}\right)$ for any finite parallelepiped $T_{0}$. By Lemma 6 the proof of Theorem 2 is complete.

\section{REFERENCES}

1. V. Beresnevich, On approximation of real numbers by real algebraic numbers, Acta Arithm., 90:97-112, 1999.

2. V. Beresnevich, On construction of regular system of points with real, complex and $p$-adic algebraic coordinates, Vesti NAN of Belarus. Ser. Fiz-mat. Nauk, 1:22-27, 2003 (in Russian).

3. V. Beresnevich, V. Bernik, and E. Kovalevskaya, On approximation of $p$-adic numbers by $p$-adic algebraic numbers, J. Number Th., 111:33-56, 2005.

4. V. Bernik, The metric theorem on the simultaneous approximation of zero by values of integral polynomials, $I z v$. Akad. Nauk SSSR, Ser. Matem., 44:24-45, 1980.

5. V. Bernik, On the exact order of approximation of zero by values of integral polynomials, Acta Arithm., 53:17-28, 1989.

6. V. Bernik and D. Vasilyev, A Khintchine-type theorem for integral-valued polynomials of a complex variable, in Proc. IM NAN Belarus, Vol. 3, 1999, pp. 10-20.

7. V.I. Bernik and M.M. Dodson, Metric Diophantine Approximation on Manifolds, Cambridge University Press, 1999.

8. N. Budarina, D. Dickinson, and V. Bernik, Khintchine's theorem and approximation of zero by the values of integer polynomials in different metrics, in Dokl. Akad. Nauk, Vol. 413.2, VSP/TEV, Utrecht/Vilnius, 2007, pp. 151-153. 
9. N. Budarina, D. Dickinson, and V. Bernik, On the exact order of approximation of zero by the values of polynomials in the fields of real, complex and $p$-adic numbers (submitted 2007).

10. A. Khintchine, Continued Fractions, University of Chicago Press, 1964.

11. E. Kovalevskaya, A metric theorem on the exact order of approximation of zero by values of integral polynomials in $\mathbb{Q}_{p}$, Dokl. Nats. Akad. Sci. Belarusi, 42:34-36, 1999.

12. V. Sprindzuk, Mahler's Problem in the Metric Theory of Numbers, Transl. Math. Monographs 25, Am. Math. Soc., Providence, R.I., 1969.

13. V. Sprindzuk, Metric Theory of Diophantine Approximation, Wiley, New York, 1979. 